\title{
Guest editorial: special issue on mobile data management
}

\author{
Dipanjan Chakraborty • Vana Kalogeraki • \\ Mohamed Mokbel
}

Published online: 19 December 2012

(C) Springer Science+Business Media New York 2012

The term Mobile Data Management has been used in a broad sense to include various aspects on the intersection of mobility and data management. The new powerful smart phones, the abundance of large rich mobile data and the coming of age of the cloud computing paradigm is opening up new avenues for research and exploration that impact larger and larger communities of humans. In this new era, the problems of seamless execution over the new computing infrastructure that encompasses smart phones, mobile sensors and cell phones, of scalable data management, of access to services, of intelligent and autonomic management of architectures, systems, algorithms and security threats, are becoming of paramount importance. In this special issue, we have made an open call for papers for research contributions related to mobile data management. In addition, we have invited the top-three papers from the proceeding of the Mobile Data Management conference, 2011, to submit an extended version to this special issue. As a result, we are pleased to present this special that includes eight excellent papers covering a wide range of topics from searching in smart phones contents to data compression in wireless sensor networks, transactions on mobile ad-hoc networks, trajectory clustering, $k$-nearest-neighbor queries, elastic cloud environment, data acquisition in smart phones, and security in a smart phone environment.

D. Chakraborty

IBM Research, New Delhi, India

V. Kalogeraki

Athens University of Economics and Business, Athens, Greece

M. Mokbel ( $\square)$

University of Minnesota, Minneapolis, MN, USA

e-mail: mokbel@cs.umn.edu 
The first paper in this issue by Konstantinidis et al., titled "Intelligent Search in Social Communities of Smart phone Users", presents a distributed search architecture, termed SmartOpt, that allows smart-phones users to search over the data of other users in a mobile social network. The main challenge is to allow efficient and effective search by minimizing the search time and at the same time minimize the energy consumption in a pure distributed environment where the data of each user are stored in the local devices. The proposed approach uses multi-objective optimization methods to generate possible routing trees for the query that try to strike a balance between these conflicting objectives.

The second paper by Szalapski and Madria, titled "On Compressing Data in Wireless Sensor Networks For Energy Efficiency and Real Time Delivery”, presents a suite of energy-efficient methods, termed TinyPack, for data compression in sensor networks. TinyPack has a high compression ratio that reduce latency, storage, and bandwidth usage further. TinyPack exploits temporal locality and delta compression to provide better bandwidth utilization important in the wireless sensor network, thus reducing latency for real time sensor-based monitoring applications.

The third paper by Xing and Gruenwald, titled "Managing Concurrent Execution of Transactions in Mobile Ad-hoc Network Database Systems: An Energy-Efficient Approach", presents an energy-efficient concurrency control algorithm for Mobile Network Databases. The algorithm is based on dynamically clustering the mobile nodes into groups and electing cluster heads that act as coordinators to commit the distributed transactions.

The fourth paper by Lei et al., titled "QS-STT: QuadSection Clustering and Spatial-Temporal Trajectory Model for Location Prediction", that addresses the problem for location prediction in mobile data services. The paper describes a framework called QuadSection clustering and Spatial-Temporal trajectory model (QS-STT) to capture movement behaviors of objects. The model first uses QuadSection clustering to extract a reasonable and near-optimal set of frequent regions. Then, it uses a spatial-temporal trajectory model that is based on a probabilistic suffix tree to explore the object's movement behavior.

The fifth paper by Zhang et al., titled "SMashQ: Spatial Mashup Framework for k-NN Queries in Time-Dependent Road Networks", presents an algorithm for Nearest Neighbor (NN) querying in a road network where the cost of traveling between two locations may dynamically change according to the current traffic conditions. The paper proposes a method to combine local and static information (e.g., locations of points of interests on a map) with remote and dynamic information (e.g., current travel times between locations). The proposed method includes a set of techniques to minimize the cost of retrieving remote information through pruning irrelevant candidates, grouping nearby data objects, and reusing recently obtained fastest routes.

The sixth paper by Nishimura et al., titled "MD-HBase: Design and Implementation of an Elastic Data Infrastructure for Cloud-scale Location Services", proposes layering a multi-dimensional index over a range-partitioned key-value store. This is achieved using Z-ordering to transform multi-dimensional location information into a one dimensional space. Then, this value is used as a key in the underlying data storage layer.

The seventh paper by Lim et al., titled "Adaptive Data Acquisition Strategies for Energy-Efficient, Smartphone-based, Continuous Processing of Sensor Streams", 
presents a framework, termed ACQUA, for 'acquisition-cost' aware continuous query processing. ACQUA utilizes a pull-based asynchronous model, where a smartphone retrieves appropriate blocks of sensor data from individual sensors, only when the stream elements are judged to be relevant to the query being processed.

The eighth, and last, paper by Li et al., titled "CAST: Context-Aware Security and Trust Framework for Mobile Ad-hoc Networks Using Policies”, proposes a contextaware security and trust framework, termed CAST, for mobile ad-hoc networks. CAST presents a quantitative model of trustworthiness for mobile ad-hoc networks where various contextual information, such as communication channel status, battery status, and weather condition, are collected and then used to determine whether the a certain misbehavior is likely a result of malicious activity or not.

Finally, we would like to extend our sincere thanks too all the reviewers for this special issue, the journal staff and Editor-in-Chief for their support and input, and to all the authors who submitted papers to this special issue. 